\title{
ENABLING DESIGNERS TO GENERATE CONCEPTS OF INTERACTIVE PRODUCT BEHAVIOURS: A MIXED REALITY DESIGN APPROACH
}

\author{
Maurya, Santosh; Takeda, Yukio; Mougenot, Celine \\ Tokyo Institute of Technology
}

\begin{abstract}
To design interactive behaviours for their products designers/makers have to use high fidelity tools like 'electronic prototyping kits', involving sensors and programming to incorporate interactions in their products and are dependent on availability of hardware. Not every designer is comfortable using such tools to ideate and test their concept ideas, eventually slowing them down in the process. Thus, there is a need for a design tool that reduces dependence on complex components of such tools while exploring new concepts for product design at an early stage. In this work, we propose a Mixed Reality system that we developed to simulate interactive behaviours of products using designed visual interaction blocks. The system is implemented in three stages: idea generation, creating interactions and revision of interactive behaviours. The implemented virtual scenario showed to elicit high motivation and appeal among users resulting in inventive and creative design experience at the same time. As a result, designers will be able to create and revise their interaction-behavioural design concepts virtually with relative ease, resulting in higher concept generation and their validation.
\end{abstract}

Keywords: Conceptual design, Experience design, Virtual reality, Mixed Prototyping, Early design phases

Contact:

Maurya, Santosh Kumar

Tokyo Institute of Technology

Mechanical Engineering - Engineering Sciences and Design

Japan

maurya.s.ab@m.titech.ac.jp

Cite this article: Maurya, S., Takeda, Y., Mougenot, C. (2019) 'Enabling Designers to Generate Concepts of Interactive Product Behaviours: A Mixed Reality Design Approach', in Proceedings of the 22nd International Conference on Engineering Design (ICED19), Delft, The Netherlands, 5-8 August 2019. DOI:10.1017/dsi.2019.199 


\section{INTRODUCTION}

The way products interact with user and environment affects their usage and acceptance rate, making the designed interactive-behaviour a crucial part of product design process as product interactions also extend to behavioural communication with users, making it feel livelier and user-centric. Products' interactive-behaviours have evolved with technology through improvements in manufacturing, materials, electronics and prototyping tools that have made the highly interactive devices present today possible. Also, the advent of easy to use mass production machines and availability of modular sensors, have reduced the cost, time and effort to develop products with detailed interactive behaviours. As a result, more makers are creating functionally interactive products. The term 'interaction' with respect to designed products, can represent interactions among the product and the user, the product and the surrounding or among all of them. Creation of such products involves thorough concept development, quick and dirty prototyping, idea selection and refinement, rapid prototyping and user testing. However, the resulting product experiences are difficult to evaluate at early phase as designers are unable to perceive new concepts that generally lack their multimodal or 3D form (Rieuf et al., 2017). This has made designers/makers heavily dependent on prototyping tools to test their ideas at early development stages. Therefore, designers need to be aware of pre-requisites required for using a prototyping tool, like its learning curve, target usage, fidelity of prototype depending on requirement, collaboration with peers and end-users, professional skills require to use the tool etc., for efficient concept generation. As stated, there are different aspects that are required to be considered while designing an interactive product. This work focuses on integrating such components in form of a Mixed Reality (MR) design tool to conceptualise new interactive product behaviours. In this work, we discuss about existing popular interaction-creation tools and establish the need for a new design tool, for which designers would be less dependent on pre-acquired skills, resources and time. The proposed tool is implemented through an empirical study over three defined stages - ideation, creation and revision - for generating interactive concepts. This implementation provides users sufficient time to explore in turn making the design activity more stimulating, so that the generated concepts result in better experience (Rieuf et al., 2017). Here we present a basic scenario for realising product behaviours and effects of user actions, and its preliminary evaluation in form of user feedback at the end.

\section{THEORETICAL BASIS AND MOTIVATION}

\subsection{User interfaces for interactions in daily life}

We experience natural and man-made interactions in our surrounding every day which can be categorised easily by arranging them based on how they are/can be perceived in their natural environment. A straightforward way to do so, is in terms of senses: vision, touch, hearing, smell and taste. Another way is in terms of their existential states: changes in physical dimensions, appearance or functionality. A basic observation of interactions based on observed daily-life scenarios is shown in Figure 1 in form of a mind map. As observed, the product-user (direct) interactions that are common in products generally focus on vision, touch and hearing senses, for example smartphones, public transport interfaces, laptops, toys etc. Indirect interactions like air conditioning device's behaviour, automatic lights and escalators etc., focus on environment-product-user type interactions. Designing for such interactions is largely treated as designing for interactive interfaces, the most popular being digital devices. A general principle to design a good interface is the principle of least astonishment (Raymond, 2003; Wahid et al., 2011), which is based on the concept that users usually pay attention to one thing at one time. This raises the bar to make such interfaces simpler irrespective of the how complex the features of the product interaction are, as seen in the case of Tangible user interfaces (TUI). By using the concept of bits and atoms (Ishii and Ullmer, 1997), intuitive interactions can be created through tangible communications involving physical objects. As physical objects are easier to relate to compared to multifunction tools like mouse and keyboard for end users, due their high affordance and relatable nature. The resulting interactions may have spatial nature and can be treated as action-reaction interfaces (more like mechanical interfaces). 


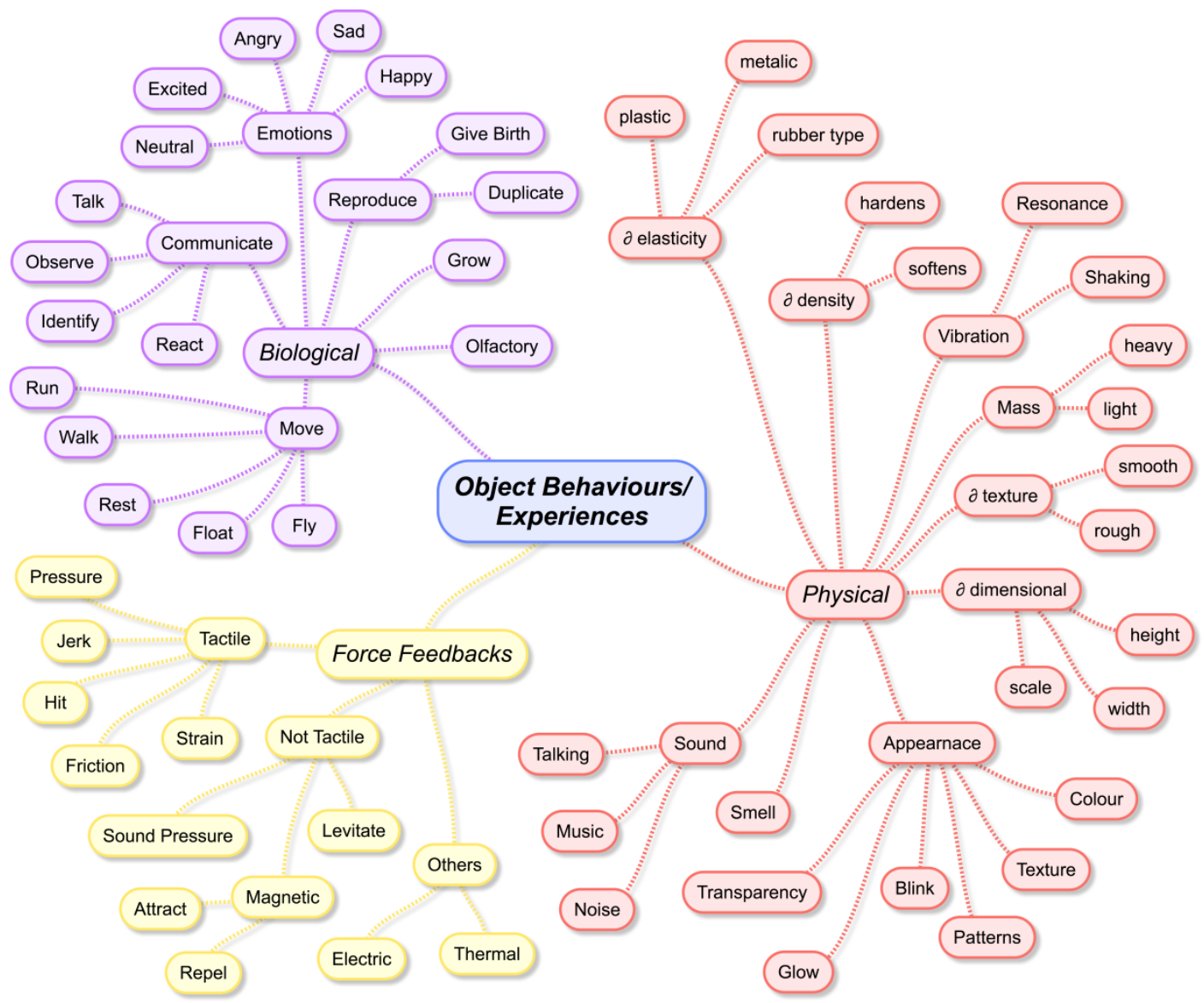

Figure 1. Categorisation of a few object behaviours observed in daily life.

\subsection{Available prototyping tools for designing interactive products}

As part of user-centred design, prototypes can enable designer to sense 'felt-life' of users for whom they are designing (Buchenau et al., 2000; Wright et al., 2008). The type of design tool being used and resulting prototypes vary depending on need, and can be classified based on their fidelity - as lowfidelity and high-fidelity types. Fidelity of a designed prototype is defined as how close it is to the look and feel of final product, and it may vary in terms of interactivity, visuals, contents and control commands. We discuss here a few tools available for creating innovative interactions for products:

\subsubsection{Lower fidelity type}

Low fidelity type tools are primitive and non-specific in nature, and are used at idea generation phase where designers try to come up with unique concepts for their products. Low-fidelity prototype result in open-discussion within the design team (Sefelin et al., 2003), the most common being pen and paper based tools. Sketching is often used at concept design stage and it lets designers to express a variety of design concepts in visual manner (Greenberg et al., 2010). Digital applications like Balsamiq (Guilizzoni, 2010), InVision and Mockplus ('Prototype faster, smarter and easier with Mockplus!', 2019) are popular among designers to create UX interactions due to quick and simple wireframe designs as they can produce low fidelity prototypes without adding complex UI designs and interactions. Inspiration Cards (Brandt and Messeter, 2004; Halskov and Dalsgård, 2006) can be of assistance in supporting concept design stage for ideation by motivating designers to explore different options. Wahid et all's (Wahid et al., 2011) toolkit of graphical elements supporting creation of design rationales and uses a similar approach. Apart from cards and sketching, designers often use catalogues, collage, clay modelling and Velcro modelling (Avrahami and Hudson, 2002; Sanders et al., 2002). Such tools have unrestricted capability to implement ideas, and resulting concepts require detailed functional specifications of the concepts in order to experience and evaluate them. 


\subsubsection{Higher fidelity type}

Higher-fidelity type tools represent more concrete information, and generate functionally-specific form of the ideas that have been expressed early in the design phase through low-fidelity tools. They are largely used to test proposed concepts by the design teams (Rettig, 1994). The resulting designs elicit higher experience and can be used for evaluating the behaviour of the product, but it takes effort to modify or redesign. Designers are increasingly adding electronic parts to increase designed prototype's functionality which in turn increases its fidelity. Based on the available resources, designer's skills and proposed concepts, they implement form and function of ideas separately, often requiring time and effort to improve the features due to increased fidelity of behavioural prototypes. The tools reducing non-designing efforts like programming and hardware connectivity, help designers to focus on idea generation and testing without restrictions. Tools like d.tools (Hartmann et al., 2006), Thumbtacks (Hudson and Mankoff, 2006), and DisplayObjects (Akaoka et al., 2010) are able to connect to computer and implement defined behaviour with relative ease. Tools like Phidgets (Greenberg and Fitchett, 2001) and the Buttercup platform, proposed by Wiethoff (2012), help designers to implement different types of sensors and effectors together as they can be intuitively and visually configured. iStuff Mobile (Ballagas and Memon, 2007) also provides visual programming platform but it is mostly aimed at programmers. Arduino is a popular example of prototyping kit addressing quick implementation and testing of ideas, due to its large community design prototypes are easy to implement with little knowledge of sensors and programming. Hence, it is easy to implement simpler behaviours but technical aspect gets complicated as designed behaviours get logically complex.

\subsubsection{Medium fidelity type}

Commercial toolkits like Lego Mindstorms and Mesh ("Sony introduces MESH - a prototype creative technology platform - Developer World", 2019) are aimed at creating more playful interactions and are relatively easy to learn. Lego Mindstorms consists of sensors and actuators that can be programmed using visual blocks making it viable at early design phases, and as seen in the work of Bissett-Johnson et al. (Ranscombe et al., 2019) with novice designers. MESH is aimed at creating interactions through visual programming and its sensors work as individual blocks capable of communicating wirelessly, generating more possibilities of applications in 3D space. Both of the toolkits are platform specific and work well with their application, but programming knowledge is required if they are connected with other tools. To visualise future products before their design is finalized Virtual Prototypes are very helpful and easily modifiable, as they are extension of computer aided design in Virtual Reality (VR) environment that can be shared with relatively ease (Bordegoni and Ferrise, 2013; Ferrise et al., 2017) keeping it at a lower fidelity level. The ability to experience the virtual designs is limited due to its intangible nature, though users can interact with it through controllers, but their ability to create or modify the design objects or parts is limited due to its unfamiliar technical nature.

\subsection{Synthesis of discussed aspects and industrial tools}

The level of fidelity can increase from low, when tools allow to test ideas using primitive resources in 2D, to high, when tools allows addition of motion, transitions and detailed behaviour (Pernice, 2016). Low fidelity tools are easier to implement and favourable for group work but mainly focus on idea generation phase. The interaction prototypes created using lower fidelity tools are good for testing ideas with users but it is far from testing the real product. On the other hand, higher fidelity tools are capable to create functional and experience-able prototypes but have a higher learning curve and less suited at early design phases. Modular toolkits mentioned are easier to use and create interactions, but they don't represent form and function appropriately at the same time. Based on the plus points of discussed tools, the ideal prototyping tool, from designers' point of view would be independent of physical parameters and easy to configure (e.g. without complex programming), and still can offer range of product behaviours which are generally a result of usage of sensors and actuators. Also, it would be essential to experience the designed product behaviour themselves instead of just observing a demonstrations (Buchenau et al., 2000). A mixed reality (MR) tool towards behaviour-ideation seems to be promising, providing higher immersion and control, through modular visual blocks that are easy to manipulate and experience. We discuss the implementation of such a tool in following sections. 


\section{PROPOSTION: IMPLEMENTED TOOL FOR QUICK CONCEPT IMPLEMENTATION OF INTERACTIVE BEHAVIOURS}

We propose usage of MR design tool for implementing quick ideation and testing of resulting product behaviours. The proposed tool assists from idea generation stage to revision of generated concept stage. It focusses on creation and testing of interactions for a given object in the virtual environment through haptic and immersive experience. For this work, we created an interaction-element catalog simulating sensor and actuator functions for product-user behaviours that act as building blocks and can be combined together to define new concepts of product behaviour. It is connected to a designed VR scenario for simulating the designed product behaviour concepts that are interact-able, share-able and iterate-able.

\subsection{Interaction-element virtual catalog and terms}

In order to ideate new interactions, the tool provides a catalog of basic elements of interactions observed in daily life. The elements in catalog serves as primitive building blocks to be used for creating an event or a set of interactive behaviours. When the catalog elements are selected during tools' usage, the virtual product demonstrates the behaviour related to the selected catalog, enabling engineers to experience the behaviours. Figure 2, shows these basic elements categorised in form of how they can be perceived through senses (vison, tactile and hearing) and devices (HMD: Head Mounted Display, TUI, and computer peripherals). For creating interactions, the virtual products are defined to have existing state (as a function of time and product behaviour) that gets altered due to a certain trigger or action (external factor). An event, here, is defined as any change occurring in the product due to stimuli like user action, time or environment. Figure 3, shows the pattern of interactive sequence creation with sample Action and State blocks. To create an interactive-behaviour conceptually, States are defined at different time intervals and Actions are defined to alter the defined states. The user is able to select elements from the virtual catalog blocks that can be implemented individually or concurrently as defined by the users.

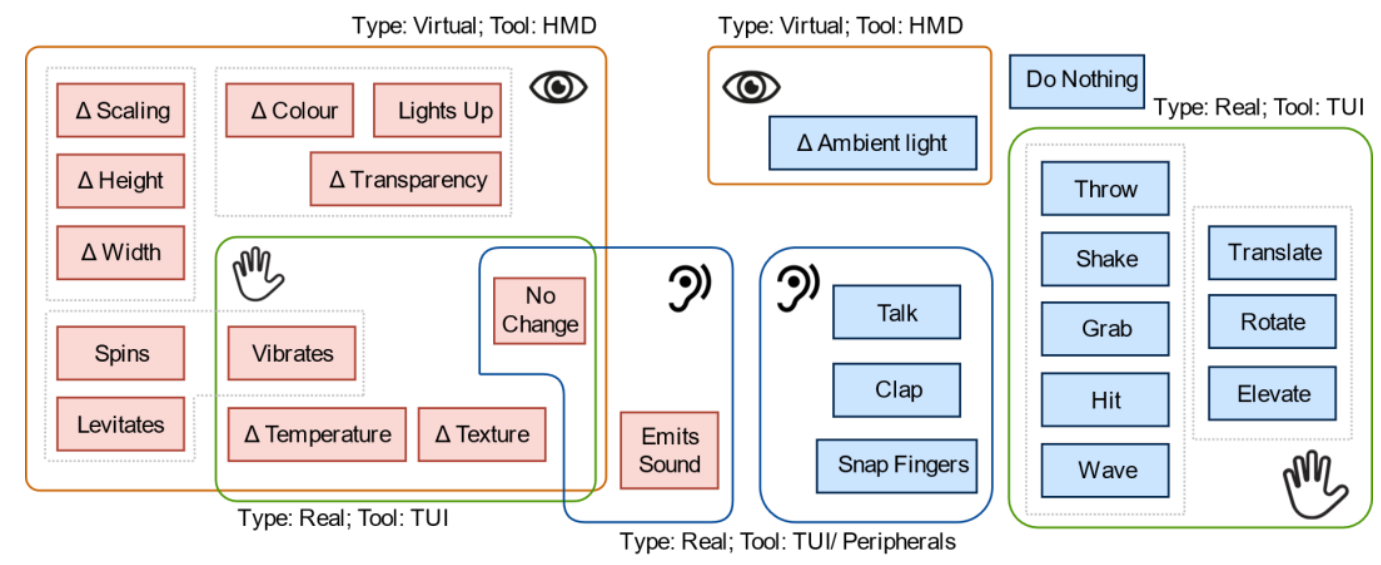

Figure 2. Categorisation of a few product behaviours (red) and user actions (blue) based on perceivability through senses and tools.

\subsection{Stages of generating product behaviour in VE: implemented protocol}

The tool provides three stages to generate concepts for interactive behaviours which lets user to focus on one aspect of concept generation at one time, as discussed below:
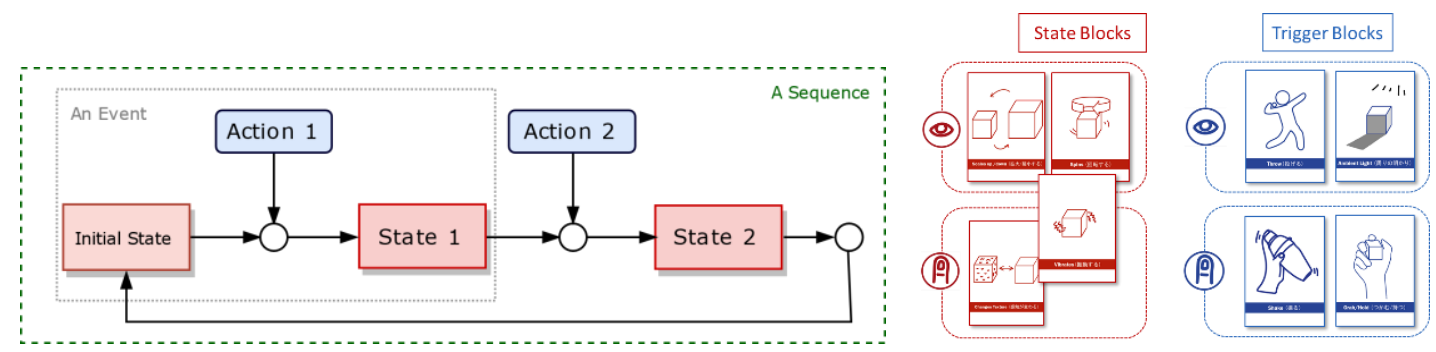

Figure 3. Creation: interaction event sequence. 


\subsubsection{Ideation: idea generation}

At ideation step, 2D animated form of catalog is shown and is used as inspiration material. Here, users think about various probable interaction behaviours to be implemented and define the set of interactive behaviours; stating how these behaviours will get executed.

\subsubsection{Creation: implementing event sequence}

At creation stage, users make the interactive ideas defined at ideation stage, using the element of catalog or building blocks by simply selecting by keyboard, mouse or hand-gestures and generate the flow of interactive behaviour or sequence, as seen in Figure 3. The created interaction behaviours are saved and are interact-able virtually any time later (demo mode). If the user is not satisfied with the experience, they can modify the created behaviours.

\subsubsection{Revision: improving functional parameters}

Here, previously created behaviours can be revised to more specific details, like modifying, replacing, removing, adding of actions/state elements in existing interaction sequences. All versions of modifications are stored as separate interaction sequences.

Parametric modification: In order to keep the accurateness of the behaviours created, in revision part users can modify the amplitude and frequency of the behaviours implemented. Amplitude modification results in change in magnitude/ strength of behaviour, for example if vibration is implemented, then its amplitude will increase. Similarly, frequency modification result in change of frequency for the implemented behaviour.

\subsection{System description and implementation}

We implemented the proposed MR design tools in 3 parts: VR environment, TUI, and workstation hardware, based on work of Maurya et.al (Maurya et al., 2019). Virtual scenarios were developed using Unity3D software. For higher immersion, the tool uses stereoscopic display through head mounted display using Oculus Rift DK2 which is installed with additional tracking sensor to monitor user's head position, and adjust the virtual scene accordingly. It provides a sense of improved presence even though it offers limited mobility options. To provide control (Slater et al., 1996) in VE, we use Leap Motion and TUI with augmented marker-tracking. Leap Motion generates a virtual representation of user's hand inside the scene, and it simulates their hand actions. The virtual representation of hand acts like a virtual controller, which is pre-programmed to simulate user-actions like selection, scrolling, throwing, drag and drop etc. TUI is tracked by an overhead webcam using markers resulting in accurate real-time imitation of motion in 3D-space to the virtual scene. It simulates a few basic (created) functional behaviours by default using sensors (piezo-electric, sound sensors, shake etc.) and with marker detection it simulates user/object behaviours like vibration, detect shaking, grab, push, clapping etc. System uses both virtual and augmented reality to provide immersion and control to the user.

\subsection{Working of MRE}

Virtual scenario created for this work is shown in Figure 4-a, it comprises of table-chair workspace assisted with catalog display (in front of the table) and user activity display (on table). Users can pick the interaction-elements from the displayed catalog screen using their hands or keyboard. The object on the table (see Figure 4-b), represents TUI object in virtual environment and animates according to selected interaction-element or manipulations by user (see Figure 4-c). Users can then finalise the selection or make a new selection. In this way they create a sequence of interaction-elements representing one concept of interactive behaviour which is also displayed on the activity display. 

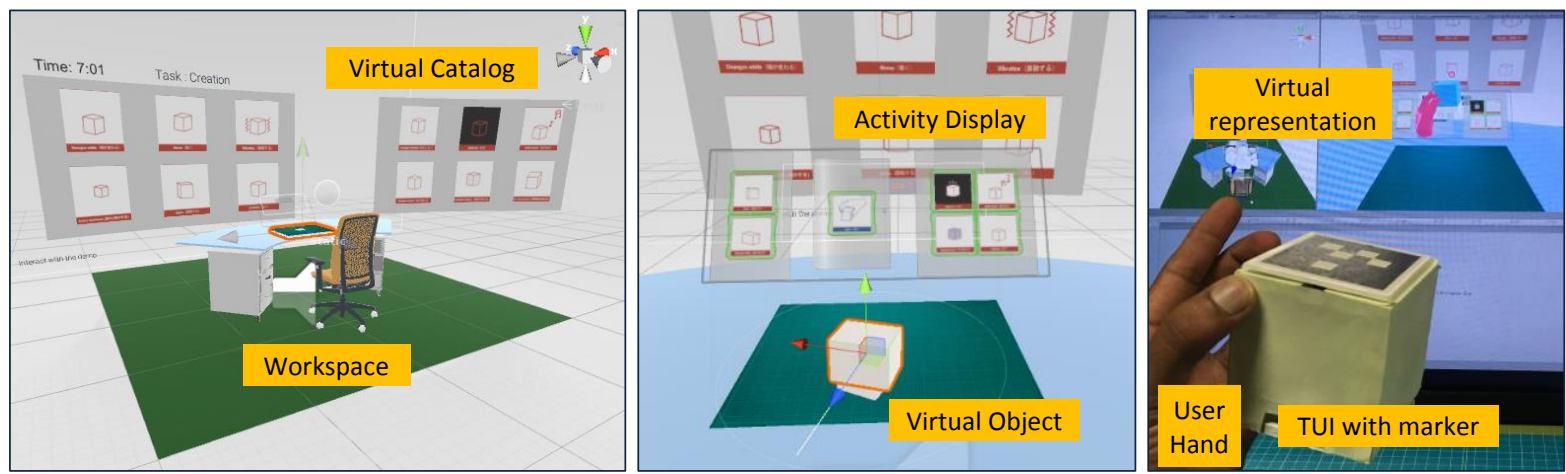

Figure 4. a) Virtual environment; b) user's view and object; c) user interacting with TUI.

This user selection-activity is automatically stored in form of sequences, represented in system as shown in Figure 5-a, where each rectangle shown represents one state at a given time (shown sequence has two States-1 and States-1, with start and end points), and each state has certain interaction catalogelements associated that were selected by user, representing the designed momentary object behaviour, see Figure 5-b (State-1 has two elements: spins and changes width; State-2 has 4 elements: lights up, emit sound, change colour (green) and levitate). Figure 5-c, shows the resulting simulation of product behaviour as mentioned in State-2 (it simultaneously lights up, changes colour, emits sound and levitates). After experiencing the behaviour, users can make changes if required to the defined sequence or revise it later.
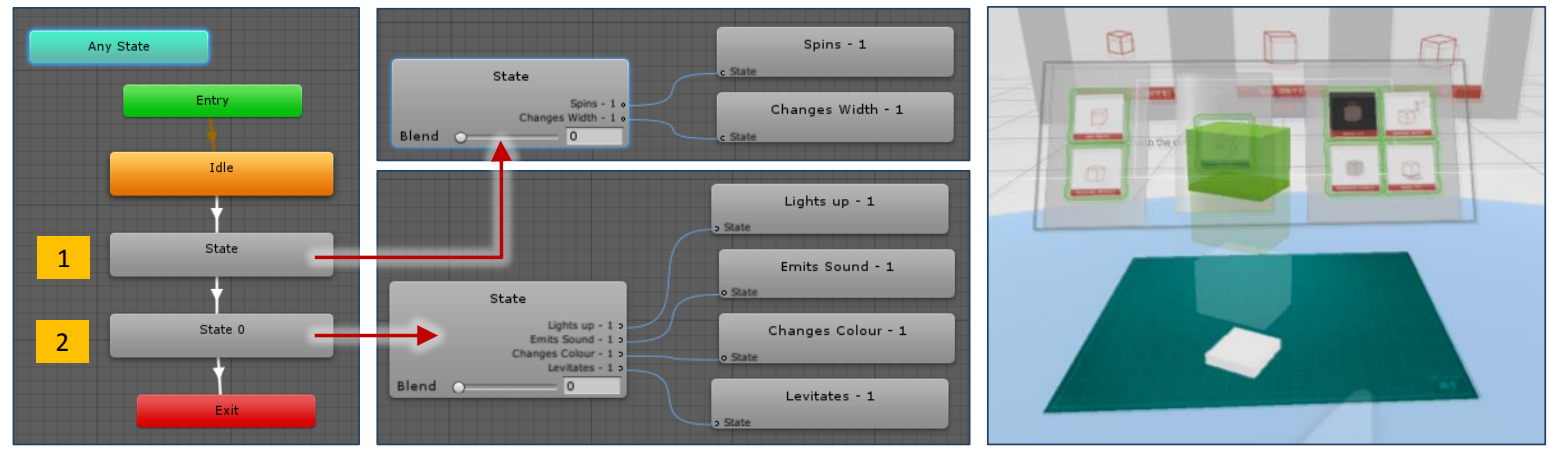

Figure 5. a) Created sequence, b) defined behaviour states, c) resulting simulated state.

\section{PRELIMINARY EXPERIENCE RESULTS}

We conducted an experimental study to evaluate the tools' design experience, with 30 participants (11 Female and 19 Male, median age $=24.17$ and standard deviation SD $=1.79$ ) having engineering design background, with self-reported experience (median values) in design and use of VR of 3.0 (SD $=1.01)$ and $2.0(\mathrm{SD}=.95)$ on a 5-point scale where 1 being novice and 5 being expert level. We asked participants to create ideas of playful interactive behaviours for given objects - alarm clock and table lamp, using proposed MR tool (MRT) and using traditional paper-based storyboard tool (PST), 30 minutes each, so that the content created can be used as blueprint or inventory for designing the real products after the task have been completed. Participants were asked to follow ideation, creation and revision stages to maximise and compare the respective outputs at each stage, Figure 6 shows the participant's activity during their respective tasks. Order of PST and MRT tasks was counterbalanced to negate effect of one task over another. After each task we collected feedback in form of questionnaires and free comments. Feedback questionnaire data focussed on the design experience participants had and their perception of the design methods/materials used during their respective tasks. It was based on selected semantics, similar to ones discussed as Product Reaction Cards discussed by Tullis and Albert (2013), in form of self-reported matrix, on a 7-point scale. It consisted of following semantics: simplicity, practicality, clarity (clearly structured or confusing), inventiveness, creativeness, challenging, pleasantness, appealing and motivation. 

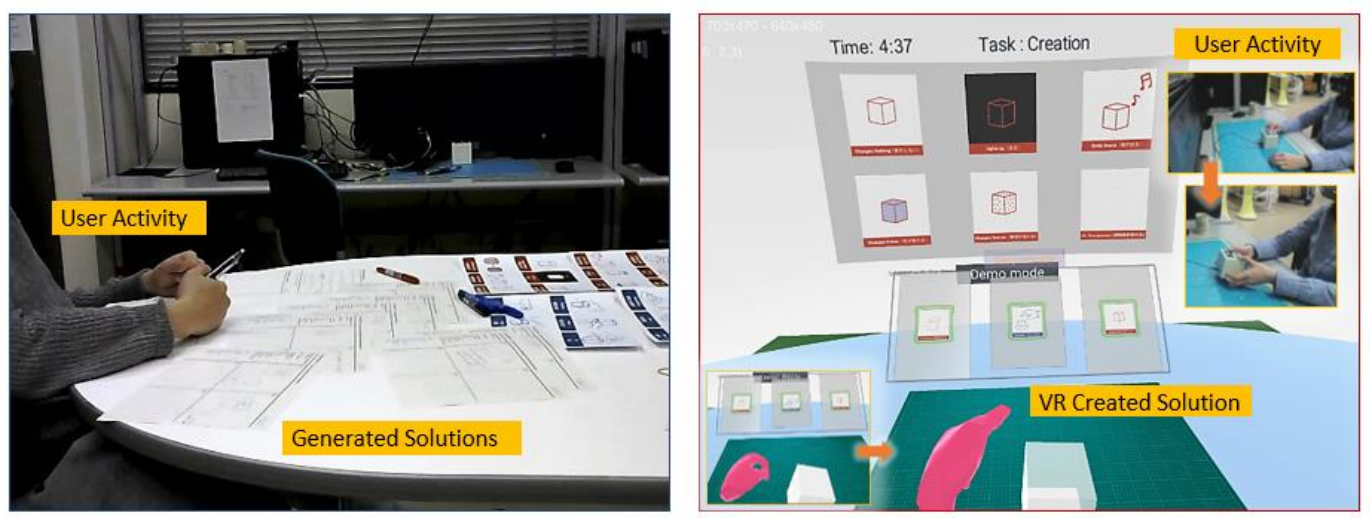

Figure 6. Participants activity during experiment: PST and MRT design tasks.

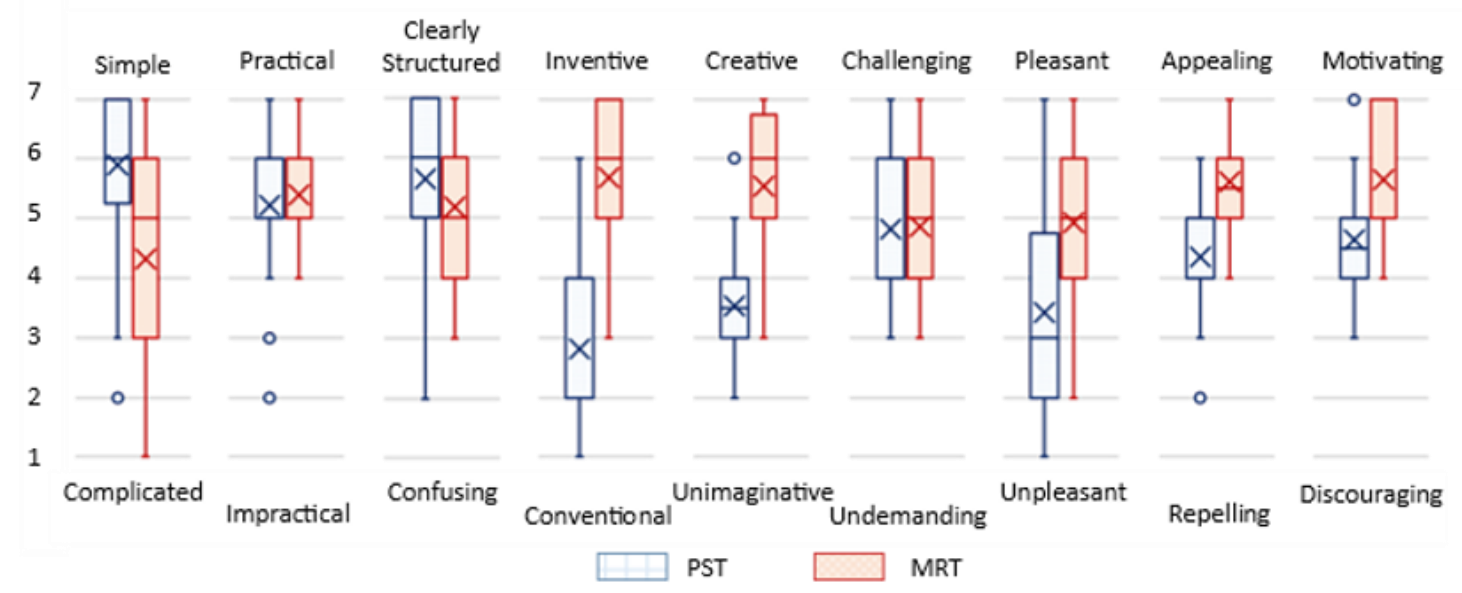

Figure 7. Design experience feedback, on a 7-point Likert scale.

Figure 7, shows the box plots of the questionnaire feedbacks. We conducted paired samples t-test between data obtained for PST and MRT design experiences, to assess relative performance relative to PST. MRT was perceived more inventive (mean of the differences $\overline{\mathrm{d}}=2.83$, $\mathrm{t}$-value $\mathrm{t}=8.43$, significant difference $\mathrm{p}=0.00)$, creative $(\overline{\mathrm{d}}=1.97, \mathrm{t}=5.77, \mathrm{p}=0.00)$, appealing $(\overline{\mathrm{d}}=1.20, \mathrm{t}=4.62, \mathrm{p}$ $=0.00)$ and motivating $(\overline{\mathrm{d}}=1.07, \mathrm{t}=3.88, \mathrm{p}=0.00)$, PST was perceived simpler $(\overline{\mathrm{d}}=-1.67, \mathrm{t}=-5, \mathrm{p}=$ $0.00)$ and undemanding $(\overline{\mathrm{d}}=1.26, \mathrm{t}=2.68, \mathrm{p}=0.01)$. We were not able to conclude participant's preference for practicality $(\overline{\mathrm{d}}=0.23, \mathrm{t}=0.80, \mathrm{p}=0.42)$, clarity $(\overline{\mathrm{d}}=-0.37, \mathrm{t}=-1.34, \mathrm{p}=0.19)$, and pleasantness $(\overline{\mathrm{d}}=0.1, \mathrm{t}=0.37, \mathrm{p}=0.72)$. Hence, we checked participants' rating for number of cases where 'MRT >= PST', 0 for false and 1 for true, and we observed significant difference for practicality (mean $\overline{\mathrm{x}}=0.3, \mathrm{t}=3.53, \mathrm{p}=0.00$ ) and pleasantness $(\overline{\mathrm{x}}=0.47, \mathrm{t}=5.03, \mathrm{p}=0.00$ ), concluding MRT to be at least as practical and pleasant as PST. We also observed positive emotions and higher excitement for MRT, as self-reported by the users indicating the effectiveness of the tool in terms of design and experience.

In free-comment users tend to report things like they were not able to create or test certain interaction elements e.g. temperature, wind blowing etc., as these were not provided as usable option for MRT for initial tests. In PST, users tend to report inability to perceive or experience the created interactionelements making it difficult for them to generate playful ideas. Users showed familiarity with traditional storyboarding (PST) but were sceptic about their creations, as reported by one user as, "...cannot specifically observe/imagine its general state from $3 D$ vision, hard to imagine the whole object......drawing will be more familiar in daily thinking, the process and refining steps will be clear and easy to embed; but we cannot give a clear image: how will it look like...".

\section{CONCLUSION}

In this paper, we discussed different prototyping design tools used for conceptualising interactive behaviours and established the need of an improved tool having lower fidelity with relatively better functional capability with a smaller learning curve. We proposed a MR design tool, based on the 
findings of discussed tools, by the combination of VR, TUI (sensors and augmented markers) for creating interact-able design concepts. This tool enables users to create and test different interactive behaviours quickly and allows them to experience the interaction in VR, as it would behave in the real scenario. Through a basic scenario implementation, we observed positive response towards MRT resulting in higher motivation and appeal to generate concepts. The proposed method of ideation, creation and revision gave users ample time and space to focus on individual aspects of creating an interactive behaviour. The key benefit of this tool, as a result of MR, is that it can help to visualise interactions which are difficult to realise through prototyping tools kits and their limitations. It gives designers relatively more freedom to explore and ideate without thinking feasibility of the behaviourconcepts. Development of such a tool is expected to allow designers to focus more on creating innovative concepts and get quicker validation of their ideas.

The tool is in testing phase, aiming at the positive effect of usage of this tool on the design process for creating creative interactive products. Presently, tool functionality is limited to behaviour creation and testing, and available interaction-elements are restricted to bare minimum (non-complex implementations). In order to keep the fidelity low and assisting user to approximate the new concepts, the range of modifications is limited. Future work will include full experimental evaluation of usability and effect on creativity for designers and the designed products. We want to look into the solutions created by the users and assess tools' impact on creativity of the design process.

\section{ACKNOWLEDGEMENTS}

We appreciate for the help provided on catalog design and discussions by Kengo Arai and Izumi Watahiki. This study was partly supported by the Japanese Society for the Promotion of Science, KAKENHI 15K16167.

\section{REFERENCES}

Akaoka, E., Ginn, T. and Vertegaal, R. (2010), "DisplayObjects", Proceedings of the Fourth International Conference on Tangible, Embedded, and Embodied Interaction - TEI '10, ACM Press, New York, New York, USA, p. 49.

Avrahami, D. and Hudson, S.E. (2002), "Forming interactivity", Proceedings of the Conference on Designing Interactive Systems Processes, Practices, Methods, and Techniques - DIS '02, ACM Press, New York, New York, USA, p. 141.

Ballagas, R. and Memon, F. (2007), "iStuff mobile: rapidly prototyping new mobile phone interfaces for ubiquitous computing", Proceedings of the SIGCHI Conference on Human Factors in Computing Systems, ACM Press, New York, New York, USA, pp. 1107-1116.

Bordegoni, M. and Ferrise, F. (2013), "Designing interaction with consumer products in a multisensory virtual reality environment", Virtual and Physical Prototyping, Vol. 8 No. 1, pp. 51-64.

Brandt, E. and Messeter, J. (2004), "Facilitating collaboration through design games", Proceedings of the Eighth Conference on Participatory Design Artful Integration: Interweaving Media, Materials and Practices PDC 04, Vol. 1, ACM Press, New York, New York, USA, p. 121.

Buchenau, M., Suri, J.F., Keane, K. and Nisi, V. (2000), "Experience prototyping”, Proceedings of the 3rd Conference on Designing Interactive Systems: Processes, Practices, Methods, and Techniques, ACM, New York, NY, USA, pp. 424-433.

Ferrise, F., Graziosi, S. and Bordegoni, M. (2017), "Prototyping strategies for multisensory product experience engineering", Journal of Intelligent Manufacturing, Springer US, Vol. 28 No. 7, pp. 1695-1707.

Greenberg, S., Carpendale, S., Marquardt, N. and Buxton, B. (2010), "Sketching User Experiences: Getting the Design Right and the Right Design: Getting the Design Right and the Right Design, Interactive Technologies", Elsevier/Morgan Kaufmann.

Greenberg, S. and Fitchett, C. (2001), "Phidgets", Proceedings of the 14th Annual ACM Symposium on User Interface Software and Technology - UIST '01, Vol. 3, ACM Press, New York, New York, USA, p. 209.

Guilizzoni, G. (2010), "Balsamiq Mockups, Balsamiq".

Halskov, K. and Dalsgård, P. (2006), "Inspiration card workshops”, Proceedings of the 6th ACM Conference on Designing Interactive Systems - DIS ’06, ACM Press, New York, New York, USA, p. 2.

Hartmann, B., Klemmer, S.R., Bernstein, M., Abdulla, L., Burr, B., Robinson-Mosher, A. and Gee, J. (2006), "Reflective physical prototyping through integrated design, test, and analysis", Proceedings of the 19th Annual ACM Symposium on User Interface Software and Technology - UIST '06, ACM Press, New York, New York, USA, p. 299. 
Hudson, S.E. and Mankoff, J. (2006), "Rapid construction of functioning physical interfaces from cardboard, thumbtacks, tin foil and masking tape", Proceedings of the 19th Annual ACM Symposium on User Interface Software and Technology - UIST '06, ACM Press, New York, New York, USA, p. 289.

Ishii, H. and Ullmer, B. (1997), "Tangible bits", Proceedings of the SIGCHI Conference on Human Factors in Computing Systems - CHI '97, Vol. 39, ACM Press, New York, New York, USA, pp. 234-241.

Maurya, S., Arai, K., Moriya, K., Arrighi, P.-A. and Mougenot, C. (2019), “A mixed reality tool for end-users participation in early creative design tasks", International Journal on Interactive Design and Manufacturing (IJIDeM), Springer Paris, Vol. 13 No. 1, pp. 163-182.

Pernice, K. (2016), "UX Prototypes: Low Fidelity vs. High Fidelity”, Nielsen Norman Group (NN/G).

"Prototype faster, smarter and easier with Mockplus!" (2019), available at: https://www.mockplus.com/ (accessed 18 March 2019).

Ranscombe, C., Bissett-Johnson, K., Mathias, D., Eisenbart, B. and Hicks, B. (2019), "Designing with LEGO: exploring low fidelity visualization as a trigger for student behavior change toward idea fluency", International Journal of Technology and Design Education, Springer Netherlands, pp. 1-22.

Raymond, E.S. (2003), "Applying the Rule of Least Surprise”, The Art of Unix Programming, Addison-Wesley, p. 525.

Rettig, M. (1994), "Prototyping for tiny fingers", Communications of the ACM, ACM, Vol. 37 No. 4, pp. 21-27.

Rieuf, V., Bouchard, C., Meyrueis, V. and Omhover, J.-F. (2017), "Emotional activity in early immersive design: Sketches and moodboards in virtual reality", Design Studies, Vol. 48, pp. 43-75.

Sanders, E., William, C. and William, C. (2002), "Harnessing People's Creativity: Ideation and Expression through Visual Communication”, CRC Press, pp. 147-158.

Sefelin, R., Tscheligi, M. and Giller, V. (2003), "Paper prototyping - what is it good for?”, CHI '03 Extended Abstracts on Human Factors in Computing Systems - CHI '03, ACM Press, New York, New York, USA, p. 778.

Slater, M., Linakis, V., Usoh, M., Kooper, R. and Street, G. (1996), "Immersion, presence, and performance in virtual environments: An experiment with tri-dimensional chess", ACM Virtual Reality Software and Technology (VRST), Citeseer, pp. 163-172.

"Sony introduces MESH - a prototype creative technology platform - Developer world", (2019), available at: https://developer.sonymobile.com/2014/05/22/sony-introduces-mesh-a-prototype-creative-technologyplatform/ (accessed 8 October 2017).

Tullis, T. and Albert, W. (2013), "Measuring the User Experience: Collecting, Analyzing, and Presenting Usability Metrics", Newnes.

Wahid, S., McCrickard, D.S., DeGol, J., Elias, N. and Harrison, S. (2011), “Don't drop it!”, Proceedings of the 2011 Annual Conference on Human Factors in Computing Systems - CHI '11, ACM Press, New York, New York, USA, p. 1571.

Wiethoff, A. (2012), "Prototyping Tools for Hybrid Interactions", lmu.

Wright, P., Wallace, J. and McCarthy, J. (2008), "Aesthetics and experience-centered design", ACM Transactions on Computer-Human Interaction, ACM, Vol. 15 No. 4, pp. 1-21. 